MECHANISMS IN ENDOCRINOLOGY

\title{
Antipsychotic medication and type 2 diabetes and impaired glucose regulation
}

\author{
Clare A Whicher ${ }^{1}$, Hermione C Price ${ }^{1}$ and Richard I G Holt ${ }^{2}$ \\ ${ }^{1}$ Research and Development Department, Tom Rudd Unit, Moorgreen Hospital, Southampton, UK and ${ }^{2}$ Human \\ Development and Health Academic Unit, Faculty of Medicine, University of Southampton, Southampton, UK
}

Correspondence should be addressed to C A Whicher

Email

clare.whicher@

southernhealth.nhs.uk

\begin{abstract}
Objective: There have been concerns about the effects of antipsychotics on weight gain and the development of type 2 diabetes (T2DM). This article aims to provide an up-to-date review on the evidence addressing this issue and the practical implications for the management of people taking antipsychotics in the context of T2DM.

Methods: We carried out searches on MEDLINE/PUBMED and the ClinicalTrials.gov website in August 2017 using the terms 'antipsychotic' and 'diabetes' or 'glucose' citing articles published after 2006 preferentially.

Results: Antipsychotics are associated with T2DM and are likely to exert a causal effect of uncertain magnitude.

Children and adolescents appear especially vulnerable to these metabolic effects; as T2DM is not common in healthy younger people, the relative risk is more apparent. Antipsychotics act on glucose and insulin homeostasis in a variety of direct and indirect mechanisms. To reduce the increasing health inequalities among individuals with mental illness screening, monitoring and prevention of T2DM is important, as is improved diabetes care in this population. Conclusion: It remains unclear whether these antipsychotic medications exacerbate an underlying predisposition to the development of T2DM or have a direct effect. Potential risks need to be weighed up and balanced between improved and lasting mental health benefits and any detrimental physical health side effects. Achieving parity of esteem between mental and physical health is a worldwide priority if we wish to improve life expectancy and quality of life in people with severe mental illness.
\end{abstract}

\section{Introduction}

The prevalence of type 2 diabetes (T2DM) has dramatically increased globally over the last 30 years alongside the growth of overweight (body mass index (BMI): $25-29 \mathrm{~kg} / \mathrm{m}^{2}$ ) and obesity (BMI $\geq 30 \mathrm{~kg} / \mathrm{m}^{2}$ ) (1). 9.1\% of adults in Europe are living with diabetes, with
T2DM comprising over $90 \%$ of cases and a further $4.8 \%$ are estimated to be living with impaired glucose tolerance (2). The Framingham Heart Study identified T2DM as a key, potentially modifiable, risk factor relating to cardiovascular disease (CVD), which remains the leading

Invited Author's profile

Richard Holt MA MB BChir PhD FRCP FHEA is Prof. in Diabetes and Endocrinology at the University of Southampton and an Honorary Consultant Physician in Diabetes and Endocrinology at the University Hospital Southampton NHS Foundation Trust. Prof. Holt's current research interests are broadly focussed around clinical diabetes but specifically encompass studies of the relationship between mental illness and diabetes and diabetes and pregnancy.

www.eje-online.org

https://doi.org/10.1530/EJE-18-0022 (c) 2018 European Society of Endocrinology Printed in Great Britain

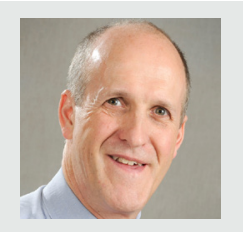

Published by Bioscientifica Ltd. 
cause of death in people with T2DM, accounting for twothirds of all deaths in those aged over 65 years (1).

People with schizophrenia and bipolar disorder, collectively known as severe mental illness (SMI), die on average 10-20 years earlier than the general population, and this mortality gap is widening (3). Much of this excess mortality is explained by increased rates of physical illness; cardiovascular disease and its risk factors occur twofold more commonly than in the general population while the rates of morbidity and premature mortality related to diabetes in people with SMI are also increased. The physical illness is increased, in part, through environmental factors, such as poor lifestyle (diets rich in saturated fat and refined sugar coupled with physical inactivity) and poverty, as well as facets of the mental illness (4). Long before the introduction of antipsychotics, there was a recognised association between SMI and diabetes (Fig. 1) and, given the longstanding link, a metabolic phenotype intrinsic to SMI has been suggested. There may also be a genetic susceptibility between the two disorders or disease-specific effects of SMI on neuroendocrine function, affecting the hypothalamic-pituitary-adrenal and growth hormone axes and inflammation.

In addition, however, health service inequalities and treatment with antipsychotics may exacerbate the situation. Antipsychotics are used worldwide for a variety of mental health disorders but the commonest licensed indication is SMI. Both conditions have a lifetime prevalence of $1 \%$ and occur equally in both sexes (5). Randomised controlled trials (RCT) have shown that antipsychotics prevent relapse and hospitalisation in

Timeline of SMI, antipsychotic medication and T2DM

- Diabetes first described

- Psychosis first defined

- Link between diabetes and pyschosis described

- First generation antipsychotics introduced

- First case report of T2DM associated with antipsychotic medication

- Second generation antipsychotics introduced

\section{Figure 1}

Timeline of SMI, antipsychotic medication and T2DM.
SMI and decrease mortality from suicide (6). Secondgeneration antipsychotics (SGA) were developed with less occupancy of dopamine D2 receptors to avoid the stigmatising extrapyramidal symptoms of first-generation antipsychotics (FGA). Since the introduction of SGAs, however, concerns have arisen that these newer agents lead to side effects of a different nature, namely, weight gain and T2DM. It is also well recognised that individuals respond differently, both in regards to efficacy and side effects, meaning the choice of treatment remains challenging.

Equivalent diabetes care in people with and without mental illness remains aspirational, and this needs to be addressed in order to improve mental health outcomes and reduce the risk of diabetes complications. There is a lack of clarity about who should take responsibility for the physical health needs of those with mental illness, and this remains a major hurdle to improving outcomes. This article aims to promote a collaborative ethos by discussing the latest evidence and guidelines and encouraging endocrinologists to reflect on their own involvement in the care of these potentially vulnerable individuals.

\section{Aims}

The aim of our review is to provide the latest evidence of the effects of antipsychotics on weight gain and intermediate metabolism, particularly in the context of the association between antipsychotic medications and T2DM. As the effects of antipsychotics on the risk of diabetes may vary according to the mental illness, we will structure our findings according to different mental health illnesses. We will initially examine the effect in SMI (adult followed by childhood and adolescents), before moving onto autism and behavioural disorders, major depressive disorder and finally dementia and delirium. We will then examine the potential mechanisms underlying this interaction and also briefly consider what other factors could be playing a role. Finally, we will discuss how management of these co-morbidities may differ and clarify physicians' specific role and responsibilities for this increasingly common scenario.

\section{Methods}

In order to write this narrative review, we searched for articles and registered trials on MEDLINE/PUBMED databases and on the ClinicalTrials.gov website respectively 
in August 2017. Searches were performed using the terms 'antipsychotic*' and 'diabetes' or 'glucose'. The additional terms 'dementia', 'delirium', 'depression' ,'autism' or 'behaviour' were also added to separate searches. We reviewed the Cochrane database but no Cochrane review has specifically examined antipsychotics and T2DM; however, glucose measurements have been included in individual antipsychotic reviews and within a review in the adolescent population. Finally, the latest guidelines addressing both mental illness and T2DM together and individually were reviewed. By necessity, we have been selective in our choice of papers, but we have concentrated on articles published after 2006 to provide the most up-todate review of this rapidly moving field.

\section{The effect of antipsychotics on glucose homeostasis}

The situation regarding T2DM and antipsychotic medications is complex not only because of the multiple confounders in people with mental illness but also because of frequent changes in antipsychotic medication in comparison to the long natural history of T2DM. In order to unpick this relationship, adequately powered prospective RCT with glucose or diabetes as a primary outcome are ideally needed. By design, the likelihood of significant bias and confounding would be reduced but no such RCTs have been reported. This is perhaps unsurprising given the length of trial that would be needed as T2DM normally develops over a number of decades and researchers' concerns around recruitment, adherence to trial protocol and retention rates in a large study design. Glucose and diabetes, however, have been described as secondary outcomes in a number of RCTs and systematic reviews. Although we can use these data, the trials are generally underpowered to examine the link between antipsychotic use and diabetes, not least because the reporting of glucose has frequently been poor.

There are several other sources of evidence that can also be considered. Case reports initially raised the question of a link between antipsychotic medications and T2DM but are limited as the findings cannot be extrapolated to the wider population. Similarly drug safety reporting is limited by a lack of a control and can be influenced by selective reporting and recall bias. Nevertheless, they may provide an indication of the size of the association.

A large number of observational pharmacoepidemiological studies and meta-analyses have linked antipsychotic medications with the development of T2DM. While these types of studies include large numbers of people exposed to antipsychotics, many fail to adjust for important confounding known risk factors for T2DM. Further limitations include the quality of clinical data and confounding by indication, namely as reports emerged about the association between T2DM and antipsychotics, it is likely that clinicians took into account perceived metabolic side effects when choosing an antipsychotic. It is, therefore, possible that drugs with a lower propensity to cause weight gain or metabolic side effects may be preferentially used in people with the highest risk of T2DM, thereby attenuating any observed difference in diabetes or glucose changes with drugs with a higher propensity to cause metabolic side effects. Despite these limitations, however, they add to the body of evidence and along with secondary outcomes from RCTs need to be considered to build a full picture.

\section{Severe mental illness}

\section{Adult population}

The literature is fairly consistent in showing rates of T2DM in people with SMI that are two to threefold higher than the general population (7). The majority of studies also suggest that antipsychotic treatment is associated with a higher prevalence of diabetes than those on no treatment $(8,9)$. One meta-analysis found the prevalence of T2DM was $2.1 \%$ in untreated people with early schizophrenia compared to $12.8 \%$ in those taking antipsychotic medication (10).

The association with T2DM appears to differ between antipsychotic agents with SGAs more strongly implicated (Table 1). A number of systematic reviews and metaanalyses of RCT have been published on this topic with one meta-analysis reporting a 32\% higher relative risk of T2DM in adults prescribed a SGA than in those on a FGA (11). A meta-analysis of head-to-head comparisons of the SGAs found olanzapine and clozapine produced a statistically significantly greater increase in glucose levels from baseline to endpoint than amisulpride, aripiprazole, quetiapine, risperidone and ziprasidone (12). In one small RCT involving 15 normal weight healthy volunteers, olanzapine showed a $42 \%$ increase in the glucose area under the curve compared to placebo during an oral glucose tolerance test (13). A recent systematic review of population-based studies also concluded that clozapine along with olanzapine are the SGAs most strongly associated with T2DM (14). A meta-analysis has also reported that the association of T2DM for olanzapine and 
Table 1 The association between treatment with antipsychotic medication and likelihood of glucose abnormalities.

\begin{tabular}{|c|c|}
\hline Antipsychotic medication & $\begin{array}{l}\text { Likelihood of treatment-related } \\
\text { glucose abnormalities }\end{array}$ \\
\hline \multicolumn{2}{|l|}{ First generation } \\
\hline Haloperidol & Lower \\
\hline Chlorpromazine & Higher \\
\hline Fluphenazine & Lower \\
\hline Perphenazine & Lower \\
\hline \multicolumn{2}{|l|}{ Second generation } \\
\hline Clozapine & Higher \\
\hline Olanzapine & Higher \\
\hline Amisulpride & Lower \\
\hline Quetiapine & Intermediate \\
\hline Risperidone & Intermediate \\
\hline Ziprasidone & Lower \\
\hline Aripiprazole & Lower \\
\hline
\end{tabular}

clozapine also appears to be independent of whether they are used in antipsychotic naive or chronic disease (RR: 1.45, 95\% CI: 1.28-1.64 for clozapine and RR: 1.29, 95\% CI: $1.20-1.37$ for olanzapine) (8).

Thirty-two Cochrane reviews have been carried out comparing various antipsychotic medications. Overall, they recognise their conclusions are based on limited data and include studies sponsored by manufacturers. One example is the review which compared risperidone and aripiprazole, at the time of the review the newest antipsychotic (Table 2). This showed that the latter had a less adverse effect on glucose metabolism (15). A metaanalysis has now also shown that aripiprazole too has a reduced risk of T2DM with odds ratios that are lower than those on no antipsychotics (OR: 0.53) (16). Nevertheless, these reviews are a useful resource for clinicians considering newer antipsychotic medications, which have not been included in older meta-analyses.

It is important for healthcare professionals and people with SMI to consider that most people taking antipsychotics will not develop diabetes. The two largest RCTs to assess the effectiveness of antipsychotics for psychiatric symptoms (primary outcome) included glucose measurements $(17,18)$. Small differences in glucose measurements were seen, but there were no reported differences in rates of T2DM. While the Clinical Antipsychotics trial of Intervention Effectiveness (17) showed an increase in glycated haemoglobin $\left(\mathrm{HbA}_{1 \mathrm{c}}\right)$, especially in those treated with olanzapine $(+0.41 \%$ ( $4 \mathrm{mmol} / \mathrm{mol}$ ) from baseline), $25.7 \%$ of participants had evidence of impaired glucose metabolism at the start of the trial as assessed by a fasting glucose concentration of $>5.6 \mathrm{mmol} / \mathrm{L}$. Arguably therefore, those with and without initial glucose abnormalities should have been analysed separately. In the effectiveness of antipsychotic drugs in first-episode schizophrenia (EUFEST) trial, the mean change in glucose concentration over 12 months ranged from 0.2 to $0.5 \mathrm{mmol} / \mathrm{L}$; no statistically significant differences between antipsychotic medications were seen (18). Dysglycaemia below the diagnosis cut off for diabetes is, however, also a risk marker for future CVD and mortality and, therefore, it is worth bearing in mind that these small changes in glucose might translate into clinical consequences in the long term.

\section{Children and adolescents}

Antipsychotic trials and reviews have overwhelmingly concentrated on the adult population, whose adverse events may not be applicable to children and adolescents. It is concerning that the relative risk of T2DM associated with antipsychotics appears to be greatest in those aged 0-24 years (OR: 8.9, CI: 7.0-11.3) (19). SGAs were associated with an elevated fasting plasma glucose levels or T2DM in $21.5 \%$ compared with $7.5 \%$ of the drug-naive group (20). While T2DM in antipsychotic-exposed youths is rare, systematic reviews and meta-analyses recognise that the relative risks are significantly higher than the general population and those not on antipsychotics (21). Some report this risk to be threefold higher (22).

Table 2 Cochrane reviews comparing various antipsychotic medications.

\begin{tabular}{|c|c|c|}
\hline Cochrane review & $\overline{\text { Studies, } n}$ & Participants, $n$ \\
\hline Aripiprazole vs placebo (2006) & 1 & 310 \\
\hline $\begin{array}{l}\text { Risperidone and Clozapine vs } \\
\text { placebo (2016) }\end{array}$ & 1 & 40 \\
\hline $\begin{array}{l}\text { Risperidone vs aripiprazole } \\
\text { (2014) }\end{array}$ & 1 & 60 \\
\hline
\end{tabular}

\begin{tabular}{l} 
Variable assessed \\
\hline Glucose $>110 \mathrm{mg} / \mathrm{dL}$ \\
$\mathrm{HbA}_{1 \mathrm{c}}>48 \mathrm{mmol} / \mathrm{mol}$ \\
Fasting glucose, MD \\
Average endpoint BG (MD; mmol/L)
\end{tabular}

Average endpoint FBG ( $\mathrm{mmol} / \mathrm{L})$

\author{
Results \\ RR: $-0.96(0.70,1.33)$ \\ RR: $-0.74(0.41,1.33)$ \\ $-4.60(-17.09,7.89)$ \\ Women: $4.29(3.97,4.61)$ \\ Men: $0.28(-0.04,0.60)$ \\ $-0.08(-0.37,0.21)$
}

BG, blood glucose; FBG, fasting blood glucose; MD, mean difference; RR, risk ratio. 
One drug safety report study simultaneously considered different age groups taking antipsychotics for a variety of indications. In this study, among those aged less than 18 years, weight gain (but not diabetes) was in the top five reported side effects in five of the seven antipsychotics examined. T2DM was the commonest side effect reported among the adult population (23).

The majority of systematic reviews in children and adolescents with SMI conclude that antipsychotic exposure time and specifically olanzapine are the main modifiable risk factors for T2DM $(21,24)$. The association between deleterious insulin resistance has also been shown especially with regard to olanzapine and United Kingdom (UK) guidance therefore recommends that olanzapine is not used first line because of its potential metabolic effects (25).

Accelerated weight gain in young individuals is a particularly serious side effect posing substantial health risks later in life (26). Pre-pubertal children run the greatest risk of alarmingly rapid weight gain although there are conflicting views as to whether disproportionately greater weight gain is seen in children and adolescents. Some authors argue that greater weight gain is not seen in this population but appears more apparent because older studies include adults who were already on antipsychotic medications and therefore were not starting from the same baseline (26).

\section{Autism and behavioural disorders}

In the paediatric population, antipsychotics are not only licensed in SMI but also in autism spectrum disorder (ASD) and Tourette syndrome. In clinical practice, antipsychotics are used even more broadly, including offlabel, in other behavioural disorders. There is a paucity of data in this area regarding their use and the development of T2DM. We only identified one article considering this topic, which supported findings seen in children and adolescents taking antipsychotic medications for psychosis. In this cross-sectional analysis, the mean concentrations of glucose and insulin rose significantly with risperidone dosage and duration implying treatment disturbed glucose homeostasis (27).

A Cochrane review in 2017 looking at SGAs for disruptive behaviour disorders in children found two trials that mentioned measuring glucose levels during treatment but no specific data were provided. Older Cochrane reviews in children with ASD did not mention glucose or T2DM but reported additional weight gain of $1.7 \mathrm{~kg}$ with risperidone and $1.13 \mathrm{~kg}$ with aripiprazole compared to placebo.
Understandably a likely relationship between antipsychotic medications and T2DM raises concerns over their use in younger populations. Whether disease factors in all psychiatric illness inherently increase the risk of T2DM is unknown. Judicious use for the shortest possible duration seems a reasonable approach given that onset of diabetes at an earlier age is likely to have greater long-term consequences.

\section{Major depressive disorders}

Compared with the general population, people with major depressive disorders also appear to have a 1.2- to 2.6-fold higher prevalence of diabetes (28). A largescale meta-analysis found that T2DM prevalence was consistently and similarly elevated in major depressive disorders, schizophrenia and bipolar disorder (11.113.7\%) compared with matched controls (29).

Epidemiological studies and studies of large samples of psychiatric populations indicate that $15-20 \%$ of individuals with major depression have psychotic features (30). Second-generation antipsychotics may be used in addition to antidepressant medication in this scenario or in drug-resistant depression. In people taking antipsychotics for depression, risk factors for T2DM include the use and duration of antipsychotic treatment, use of mood stabilisers, younger age and residence in urban areas (29, 31). However, a recent systematic review suggests that the risk from antipsychotics is small (32). In a doubleblind RCT of olanzapine plus sertraline vs olanzapine and placebo for psychotic depression, participants from all age groups experienced significant increases in weight (45.1-65.0\%) while fasting blood glucose (FBG) levels increased significantly among younger adults. These metabolic changes are consistent with those reported during olanzapine treatment among younger adults with schizophrenia, but the weight gain may be due to recovery of weight lost during the depressive episode (30).

Antidepressants, such as amitriptyline and mirtazapine, and mood stabilisers, such as lithium and valproate, have also been associated with weight change. While weight gain is generally milder, one systematic review suggests clinically relevant differences after 6-12 months. Mirtazapine, paroxetine and amitriptyline had most weight gain while bupropion was associated with weight loss (33). Antidepressants may also have an impact on mental illness-related factors relevant to the risk for T2DM, such as physical activity and diet.

While disentangling a drug effect is again challenging, it appears that awareness by healthcare professionals of the 
importance of prevention, early detection and adequate treatment of diabetes is also relevant in all people with major depressive disorders.

\section{Dementia and delirium}

Despite a lack of evidence of their effectiveness and safety concerns around increased mortality and cerebrovascular accidents, antipsychotics are also used to treat psychosis and persistent agitated behaviour in dementia and, less commonly, delirium. The United Kingdom recommends their use only after a number of stringent conditions have been met (34). Particular caution is advised with FGA in Lewy body dementia as individuals are at particular risk of severe adverse reactions. Treatment should be time limited and regularly reviewed (at least every 3 months).

The metabolic adverse effects of antipsychotics, including dysglycaemia and weight gain, in this older population are less studied but appear to be less marked. A recent systematic review in this population included 16 drug-placebo RCTs and only four reported significant weight gain (35). Hypotheses for this include an earlier peak in the risk of developing T2DM and weight loss that is commonly seen in advanced dementia, particularly in nursing home populations. As this was the setting for many of the larger scale studies (median duration of stay 5.3 months in one study (36)), this reduced metabolic risk may not be similar for people living with dementia in the community.

Few studies have reported detailed information on hyperglycaemia or T2DM but an extension of the original CATIE trial examined efficacy and safety of SGAs in 421 outpatients with Alzheimer's dementia. Reassuringly, this found no adverse effect on glucose despite clinically significant weight gain in women taking SGAs for more than 24 weeks. Compared to those not taking antipsychotics, the odds ratio for weight gain was 3.38 (95\% CI: 1.24-9.23) (37). Whether this weight gain could ultimately affect glucose metabolism is unknown.

While there are insufficient data to make definite conclusions, metabolic effects appear to be less concerning in older people, but this issue must be closely reviewed.

\section{Epidemiology conclusion}

In summary, there appears to be a link between the use of antipsychotics and the development of diabetes. Children and adolescents appear especially vulnerable to these metabolic effects while in the older population these effects are not prominent. The overall absolute risk of antipsychotics and T2DM, however, is small, and it is likely that the drugs may accelerate the presentation of diabetes rather than precipitate the disease de novo in most cases.

\section{Mechanism}

If a biologically coherent mechanism between antipsychotics and T2DM exists, this would support a causal relationship. While no specific link has yet been pinpointed, different hypotheses have been postulated, including the effect of antipsychotic-induced weight gain on insulin resistance, a direct effect on insulin signalling and a direct cytotoxic effect on the pancreatic $\beta$-cell.

\section{Weight dependent}

The association of obesity and T2DM is well established with each additional $1 \mathrm{~kg} / \mathrm{m}^{2}$ in BMI increasing the risk of T2DM by $8.4 \%$ (38). Antipsychotics are among the most obesogenic medications and this indirect route is coherent with our understanding of the pathophysiology of the development of T2DM. Weight gain is the commonest side effect of antipsychotics although to a lesser extent with FGA. Greater than 7\% weight gain from baseline has been seen in up to $72 \%$ of those taking SGAs (39). This often occurs within 8 weeks of drug initiation, especially in younger or antipsychotic naive individuals but can continue, albeit at a slower rate, for up to 4 years (18). Adiposity levels during antipsychotic treatment have been separately confirmed to be strongly related to insulin resistance (40). As, in the general population, however, T2DM is not inevitable in everyone who gains weight and we are not aware of any prospective study that shows a clear relationship between antipsychotic related weight gain and incident T2DM.

There is a hierarchy of effect on weight with certain medications (olanzapine and clozapine) being most strongly associated with weight gain, risperidone and quetiapine having an intermediate effect on body weight while aripiprazole and ziprasidone have the least effect. In children and adolescents roughly the same hierarchy for risk of weight gain with these agents has been identified (26). At all ages, however, there is also considerable variation between individuals (for example, the 5 th to 95th centile for weight change is $1.4-9.5 \mathrm{lb} / \mathrm{month}$ with olanzapine (41)) and so no antipsychotic should be considered truly weight neutral. Weight gain, however, should be considered when choosing antipsychotic medication. 
A number of hormones, neurotransmitter receptors and neuropeptides have been implicated in the mechanism of antipsychotics induced weight gain but those that regulate appetite stimulation and consequently increased food consumption are thought to be more specifically involved. These have been considered in the following basic science studies.

\section{Hormones}

Leptin is disproportionally high in relation to adiposity in those taking antipsychotics (42) which raises the possibility that the leptin signalling mechanism may be disrupted by antipsychotics. This would be consistent with the increased appetite experienced by people taking antipsychotics despite significant weight gain (43).

Serum prolactin is known stimulate $\beta$-cell proliferation, insulin production, and insulin secretion during the second half pregnancy and epidemiological studies have shown that serum prolactin is inversely associated with the risk of diabetes (43). Hyperprolactinaemia is a wellrecognised side effect of FGA, risperidone and amisulpride while the incidence of hyperprolactinaemia is much lower with the other SGA. It has been postulated that this may partly explain the difference in the risk of diabetes between FGA and SGA. By contrast, aripiprazole, which is a partial D2 receptor agonist and has the lowest rate of hyperprolactinaemia of all antipsychotics and also has a low propensity for diabetes (44).

There has also been interest that clozapine related T2DM is mediated through reduced glucagon like peptide 1 (GLP-1) raising the possibility that GLP-1 receptor agonists (GLP-1RA) could be co-administered with antipsychotics to prevent development of T2DM.

\section{Neurotransmitters}

A key receptor postulated to mediate homeostatic and hedonic aspects of feeding is the histamine $\mathrm{H} 1$ receptor, which reduces food intake (45). This is blocked by many SGAs but clozapine and olanzapine have the highest affinity. The anorexigenic serotonin $5-\mathrm{HT}_{2 \mathrm{c}}$ receptor has also been implicated (45). While both clozapine and olanzapine are potent $5-\mathrm{HT}_{2 \mathrm{c}}$ receptor antagonists and deletions of the gene for this receptor in mice results in obesity, other SGAs associated with less weight gain, such as ziprasidone, also have a higher affinity for this receptor. Antipsychotics bind to the adrenergic B3 and A1 receptors and, while the affinity for these receptors is weak, there is correlation between antagonism of this receptor and weight gain for several antipsychotics.

\section{Neuropeptides}

Neuropeptides, such as Melanocortin 4 receptor and Brain Derived Neuropeptide Factor, play a role in weight regulation. Antipsychotics increase expression of the latter in the hippocampus and prefrontal cortex and may play a role in antipsychotic-induced weight gain (42).

\section{Weight independent insulin resistance}

An alternative explanation is that antipsychotics directly cause T2DM through increased insulin resistance independent of changes in BMI, impaired $\beta$-cell function or both. This is an important area to consider as a number of people develop T2DM while taking antipsychotics without weight gain and these medications have also been associated with potentially fatal diabetic emergencies.

A unifying property of all antipsychotics is their blockade of dopamine D2 and D3 receptors. Dopamine plays a role in central glucose regulation and D2 and D3 receptors are also found in beta cells. Through negative feedback, dopamine inhibits further insulin release, and chronic insulin secretion may promote insulin resistance similar to hyperinsulinaemia in T2DM (46). In T2DM, insulin resistance may contribute to the exhaustion of $\beta$-cells.

The hypothesis that antipsychotics provoke glucose and insulin disturbances independent of weight gain is also supported by the clinical trial of olanzapine in healthy volunteers that was discussed earlier. A further clinical trial showed increased fasting insulin resistance levels in hospitalised patients taking olanzapine over 5 months compared to risperidone, despite no significant weight change. There were no differences between C-peptide levels or measures of insulin secretion (47). Marked acute hepatic insulin resistance has also been demonstrated with intravenous olanzapine in healthy rats (48).

\section{Insulin secretion}

A recent systematic review reported 72 cases of antipsychotic associated diabetic ketoacidosis (DKA), a state of marked insulin depletion (49). The review found associated weight gain in only half of the reported cases and DKA was the first clinical presentation of diabetes in the majority. More than half were associated with 
polypharmacy. Autoantibodies were only measured in 13 cases but were negative in $85 \%$, supporting the argument that these are generally not new cases of autoimmune type 1 diabetes and a direct toxic effect of the antipsychotic should be considered.

Antipsychotics act on multiple receptors that are found both in the brain and the islet $\beta$-cells and could affect insulin secretion. Antagonism of the muscarinic M3 receptor is one such example (45). One RCT concluded that amisulpiride but not olanzapine appears to acutely increase pancreatic insulin secretion in healthy individuals and that stimulation of $\beta$-cells could be a protective factor against the development of T2DM. Antipsychotics have partial $5 \mathrm{HT}_{2 \mathrm{c}}$ properties; full agonists have been shown to reduce insulin secretion in isolated islets in animal studies (48). Finally in vitro effects have suggested clozapine has a direct effect to inhibit glucose-dependent insulin release by $\beta$-cell membrane hyperpolarisation (50).

In summary, it is becoming clearer that antipsychotics act on glucose and insulin homeostasis in a variety of direct and indirect mechanism. It seems likely these complex interactions, including a direct effect on the $\beta$-cell, along with others not yet recognised, may lead to the observed changes in glucose in some individuals.

\section{Management}

To reduce the increasing health inequalities among individuals with mental illness, screening, monitoring and prevention of T2DM are important components of the physical health care of those taking antipsychotics. This has been acknowledged in numerous national and international guidelines (51).

While the fundamental principles of many of the guidelines are in agreement, there are some areas where recommendations differ. Conflicting views on both clinical matters and ambiguity over roles (i.e. who should do the monitoring) can result in inertia. A review of UK psychiatry healthcare professionals found that $17 \%$ thought it was not part of their role to provide advice about weight (52) despite national guidance stating that 'the secondary care team maintains responsibility for screening and monitoring metabolic risk factors for the first 12 months or until the condition has stabilised whichever is longer' (25). Reasons from mental health professionals for this discrepancy included $57 \%$ being worried about treating obesity and T2DM and a lack of incentive. Education of the mental health team should therefore not be forgotten. Many healthcare professionals may be unfamiliar with the notion of metabolic risk and the importance of assessing and treating this and so diabetes services should consider how to provide a rolling education programme. Appropriate agreement about clinical responsibility is needed to ensure joint working across mental and physical as well as primary and secondary care teams.

\section{Screening and monitoring}

Opportunistic screening of established and modifiable risks for the development of T2DM is important for people with SMI. In the UK, the first joint diabetes and psychiatry guidelines were published earlier this year for psychiatric inpatient settings and discuss the importance of taking this opportunity for screening, if not already performed in the community (53). A repeat metabolic risk assessment is also universally recommended if individuals are switched from one medication to another.

With the effort to reduce the use of antipsychotic medications in dementia and delirium, there has been little focus on monitoring or screening for these individuals but some groups recommend assessing cerebrovascular risk factors before antipsychotics are offered to an individuals with dementia (34).

Thorough evaluation is essential to identify those at greatest risk of metabolic side effects and to plan appropriate monitoring and therapy. Initial evaluation should ideally include pre-treatment screening as well as education. Medical history should include personal and family history of obesity and diabetes and other nonmodifiable risk factors for diabetes such as age, gender and ethnicity. Clinicians should also enquire about lifestyle, diet and exercise history. In this review, we will now consider measurements of overweight or obesity and glucose measurements but other cardiovascular risk factors such as blood pressure and lipids should not be forgotten nor smoking cessation advice.

Some guidelines recommend using a specific diabetes risk score assessment tool, such as the QDiabetes risk calculator or the Leicester practice risk score (54). Others, however, worry that these tools were designed for adults over the age of 50 years and given the concerns about risk of T2DM in children and adolescents as well as young adults, believe a more specific SMI validated risk score is needed for this population (55). Guidelines, which focus on children and adolescents, advocate that primary prevention of overweight and obesity should be the highest priority in this age group (56). 
International guidelines recommend continued monitoring of weight and glucose measurements during treatment with antipsychotic medications to evaluate for changes although the frequency varies. While measurement of waist circumference provides a better indication of adiposity linked to cardiovascular disease, it is generally felt to be intrusive and may be less practical in psychiatric settings. The European consensus statement (55) suggests that the frequency of testing will depend on the person's history and prevalence of risk factors, but a pragmatic approach to opportunistic screening may be needed (Table 3).

The recent British Association for Psychopharmacology (BAP) guidelines advise using fasting or random blood glucose measurements initially and at 12 weeks later but $\mathrm{HbA}_{1 \mathrm{c}}$ in the longer term (57). This is to avoid false negative $\mathrm{HbA}_{1 \mathrm{c}}$ results shortly after initiation if glucose levels rise sharply. While many other guidelines recommend the use of FBG in the longer term, this is likely because they were published at a time when FBG was seen as the 'gold standard' prior to the World Health Organisation inclusion of $\mathrm{HbA}_{1 \mathrm{c}}$ as a diagnostic test for diabetes. $\mathrm{HbA}_{1 \mathrm{c}}$ has the advantage of not requiring fasting, which can be challenging in this group, and is therefore generally considered appropriate. The fact that $\mathrm{HbA}_{1 \mathrm{c}}$ may not detect all glucose abnormalities, however, should be remembered (58). If symptomatic, fasting or random blood glucose measurements should be taken. Finally while the use of simple finger prick tests is sometimes advocated for simplicity, any results outside the normal range must be confirmed by laboratory testing.

Pragmatic recommendations such as 'monitoring may be carried out less frequently once weight has stabilised, but closer monitoring of weight may be required in those gaining weight' seems reasonable. All people taking antipsychotics should also be encouraged to monitor their own weight and report any weight change to their treating clinician. In order for people to seek advice and testing to confirm a diagnosis of diabetes, the importance of education concerning the acute symptoms of diabetes cannot be underestimated. Once established on medication, quarterly, biannually or annually measurements of weight and $\mathrm{HbA}_{1 \mathrm{c}}$ are advised depending on the guideline.

It is recognised that screening and monitoring is often not being done in routine clinical practice. A recent audit in the UK found that only $56 \%$ of people with schizophrenia had a record of blood glucose control (52). This figure was co-incidentally identical in a meta-analysis of studies examining routine metabolic screening practices in those taking antipsychotics in five countries $(56.1 \%$ (95\% CI 32.4-63.7)) (59). In a number of cases, local or national guidelines were then implemented and direct head-tohead pre- and post-guideline implementation showed a modest but significant (15.4\%) increase in glucose testing. Although guidelines can increase monitoring, most people still do not receive adequate testing.

\section{Prevention of diabetes}

All people with SMI and their carers should be made aware of the risk of weight gain and T2DM. This is especially important for people who are overweight or obese at the start of therapy or have a family history of obesity or diabetes. Giving quantitative estimates of expected weight gain with different antipsychotics can help individuals make an informed decision about treatment (53).

Most guidelines recommend advice on physical activity, diet, psychoeducation of the individual and their family, and referral for advice and treatment. These should aim to help someone who is overweight or obese to achieve and maintain a $5-10 \%$ weight loss and progress to a healthy weight. Advice should be simple and focussed on the importance of diet and exercise in preventing initial

Table $3 \mathrm{HbA}_{1 \mathrm{c}}$ screening and consequent management advice for asymptomatic people on antipsychotic medication.

\begin{tabular}{l} 
Point of antipsychotic \\
treatment \\
\hline Before starting \\
antipsychotic \\
First year on \\
antipsychotic \\
Established on \\
antipsychotic \\
Changing \\
antipsychotic \\
regimen
\end{tabular}

\begin{tabular}{|c|c|}
\hline $\begin{array}{l}\text { When and what glucose } \\
\text { measurements to check }\end{array}$ & 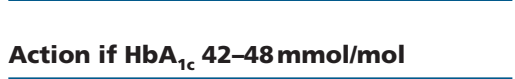 \\
\hline $\begin{array}{l}\text { Fasting or random blood glucose } \\
\text { before initiation } \\
\text { 1. Fasting or random blood } \\
\text { glucose at } 3 \text { months } \\
\text { 2. } \mathrm{HbA}_{1 \mathrm{c}} \text { at } 12 \text { months } \\
\mathrm{HbA}_{1 \mathrm{c}} \text { annually if previous result } \\
<42 \mathrm{mmol} / \mathrm{mol} \\
\mathrm{HbA}_{1 \mathrm{c}} \text { at } 3 \text { and } 12 \text { months after } \\
\text { change then as per established }\end{array}$ & $\begin{array}{l}\text { 1. Intensive lifestyle intervention. } \\
\text { 2. Ensure been screened and offered } \\
\text { intervention for other CV risk } \\
\text { factors } \\
\text { 3. Repeat } \mathrm{HbA}_{1 \mathrm{c}} \text { in } 6 \text { months }\end{array}$ \\
\hline
\end{tabular}

Action if fulfils criteria for T2DM diagnosis

1. Treat as per local T2DM guidelines

2. Refer for T2DM education

3. Ensure enrolled in local screening services

4. Ensure been screened and offered intervention for other $\mathrm{CV}$ risk factors 
weight gain, as subsequent weight loss is more difficult to achieve. Providing information on local facilities for exercise and physical activity or relevant support groups and weight management groups should be made available to individuals who are already overweight. Advice should also consider lifestyle and dietary issues common in individuals with schizophrenia (diet rich in saturated fat and refined sugar). Only $58.2 \%$ of people recently asked, however, felt they were given personalised advice about diet and nutrition (52). The importance of behaviour change should be frequently emphasised to enable this to be put into practice.

While lifestyle interventions are the cornerstone for prevention of T2DM, many mental health trusts struggle to implement this. This may be because it is unclear how these should be delivered in this specific population. Evidence-based programmes, such as the Diabetes Prevention Programme (DPP), did not included individuals with SMI. Although no lifestyle diabetes prevention trials have been undertaken in people with SMI, a number of studies have assessed the effect on body weight. A meta-analysis of 17 small studies has indicated that non-pharmacological interventions lasting $12-16$ weeks lead to a mean weight reduction of $3.12 \mathrm{~kg}$ (60). A more recent meta-analysis of interventions lasting longer than a year, however found significant weight loss in only two of the six included studies (61).

Two long-term trials from the US have shown that intensive lifestyle management over a year can achieve significant weight loss. The ACHIEVE study studied the effect of combined group weight management sessions (weekly in the first 6 months then monthly), monthly individual visits and thrice weekly group activity classes in 291 people attending community psychiatric outpatients (62). The intervention resulted in a mean weight loss of $-3.2 \mathrm{~kg}$ over 18 months. The more recent STRIDE study found a $4.4 \mathrm{~kg}$ weight reduction in intervention participants compared to usual care after 6 months but this difference fell to $2.6 \mathrm{~kg}$ by 12 months when the intensity of the intervention was reduced (63). In both of these studies, significant numbers of the participants had mental illness other than schizophrenia spectrum disorders, for whom behaviour change may be easier to achieve. The importance of these diagnostic differences may explain why the Danish CHANGE study, which randomised 428 people with schizophrenia spectrum disorders and abdominal obesity to 12 months of intensive lifestyle coaching plus care coordination, or care coordination, or usual care alone found no reduction in body weight or waist circumference with either intervention (64).
The optimal weight management of people with SMI remains uncertain but is likely to include a multimodal approach which includes individually tailored lifestyle advice, optimisation of antipsychotic medication and adjuvant therapies such as metformin (57).

\section{Prediabetes}

While the numerical cut off at which one has 'prediabetes' remains controversial, it is well accepted that there is a group of the population at high risk of developing diabetes. Closer monitoring of weight and glucose measurements, combined with intensive advice and support on diet and exercise is generally recommended.

In addition to lifestyle interventions, metformin has been shown to reduce the incidence of T2DM in the general population. Systematic reviews of metformin and antipsychotic related weight gain found metformin reduced weight by a mean of $-3.17 \mathrm{~kg}(95 \% \mathrm{CI}-1.90$ to -4.4) (65). Greater weight loss, however, is likely to be needed to prevent T2DM. A double-blind clinical trial of metformin vs placebo in combination with antipsychotics with a primary outcome of glucose measurements $\left(\mathrm{HbA}_{1 \mathrm{c}}\right.$ and OGTT) is currently recruiting. While metformin is general safe, it is not appropriate for individuals with alcohol dependence syndrome which is common in people with SMI (20.6\% lifetime risk) because of the risk of lactic acidosis.

Other approaches have been considered more recently and two trials have assessed the effects of GLP1RAs in people taking antipsychotics. In the first, onceweekly exenatide did not promote weight loss (66) but improvements in both body weight (placebo subtracted $5.3 \mathrm{~kg}$ ) and glucose measurements were seen in a larger $(n=103)$ using once daily liraglutide. $63.8 \%$ with prediabetes reverted to normal glucose tolerance (67).

In clinical practice, switching antipsychotic is common; however, evidence for an effect on glucose metabolism is limited. While there are cases of T2DM which remit on withdrawal of antipsychotics, this is not generalisable to the population as a whole. Situations where this approach may be appropriate, include people who experience a $>5 \%$ increase in body weight from baseline or worsening glycaemia. Antipsychotic medications should be cross-titrated gradually and abrupt withdrawal should be avoided. Particular caution should be taken when considering withdrawal of clozapine because of the potential for serious psychological sequelae. Another strategy considered by the BAP guidelines was the addition of an antipsychotic with a perceived lower 
diabetogenic potential (57). The addition of aripiprazole to clozapine and olanzapine led to a $2 \mathrm{~kg}$ weight loss but glucose effects were inconsistent (68). The degree of polypharmacy in this scenario must also be considered.

The most effective and cost-effective methods for identifying, assessing and managing the risk of T2DM among high-risk, vulnerable adults remains an important research question.

\section{Management of diabetes}

T2DM is initially often asymptomatic and it is estimated that up to $70 \%$ of cases in people with SMI are undiagnosed. However, appropriate monitoring should establish this diagnosis and guidelines generally advise a referral to the general practitioner or local diabetes team. The management of T2DM in people taking antipsychotic medication should follow the same principles and guidance of the general population with T2DM (69). In addition, it is worth considering a multidisciplinary team care approach as it is recognised that people with SMI often benefit from this. Medications that may contribute to weight gain should be avoided where possible while newer agents, such as GLP-1RAs and sodium glucose cotransporter 2 inhibitors (SGLT2), may be appropriate. Cardiovascular risk should be aggressively managed along with an annual assessment to review and screen for early signs of retinopathy, neuropathy and diabetic nephropathy.

Consideration of antipsychotic medication choice may be reviewed again. Although switching to an antipsychotic with a lower propensity to increase glucose concentration may help, given the heterogeneity of dysglycaemic potential and weight gain among individuals this is by no means a guaranteed solution.

While there is agreement that people with T2DM taking antipsychotics should have access to the same high quality care as the general population making this a reality is more challenging. Less than half of those with SMI and T2DM met the glycaemic control target of $\mathrm{HbA}_{1 \mathrm{c}}<58 \mathrm{mmol} / \mathrm{mol}(7.5 \%)$ and their risk of diabetic complications is increased (54). Care pathways for people with mental health problems and T2DM are often fragmented which builds barriers for people with SMI (52). Stigma around mental health remains an issue and it is recognised that individuals with psychiatric disorders often struggle to access routine physical healthcare or establish timely contact for acute issues as a result of both mental health and organisational reasons. The ability for healthcare providers to share core information is often lacking.
Weight gain and a diagnosis of T2DM can also reinforce an individual's negative view of themselves and unsurprisingly individuals wish to stop the implicated medication (52). This is a significant concern as mental health needs are more likely to be adequately met if people with SMI are taking antipsychotics (70) and good mental health is essential to enable individuals to make the daily self-management decisions which are necessary in T2DM. It is also less likely that lifestyle management programmes will be successful if individuals are acutely psychotic.

\section{Conclusion}

People taking antipsychotics are at increased risk of T2DM. Although these medications appear to increase the risk, these potential adverse effects need to be balanced against the improved and lasting mental health benefits. It is important that people with SMI are not denied effective treatment without good reason and individual factors must be considered in each case. Nevertheless, greater attention to the possible impact of antipsychotics on the physical health of people is needed. More research to develop and evaluate novel clinical innovations to prevent T2DM and better support those with diabetes and mental illness is needed. People with mental illness should be included in appropriate trials to reduce the risk of widening health inequality.

Diabetes and mental illness can both be challenging lifelong conditions but opportunities exist for healthcare professionals to improve the current situation for this potentially vulnerable and high-risk group. Key areas include improved integrated care pathways and communication, specialist training for healthcare providers and commissioning to incentivise best practice. Achieving parity of esteem between mental and physical health is a worldwide priority if we wish to improve life expectancy and quality of life in people with SMI. 'Death in old age is inevitable, but death before old age is not'. (Richard Doll)

Declaration of interest

R I G Holt received fees for lecturing, consultancy work and attendance at conferences from the following: Boehringer Ingelheim, Eli Lilly, Janssen, Lundbeck, Novo Nordisk, Novartis, Otsuka, Sanofi, Sunovion, Takeda, MSD. H Price received fees for lectures, advisory boards and attendance at conferences from Novo Nordisk.

\section{Funding}

This research did not receive any specific grant from any funding agency in the public, commercial or not-for-profit sector. 


\section{References}

1 WHO. Diabetes factsheet. World Health Organisation. (available at: http://www.who.int/mediacentre/factsheets/fs312/en/)

2 International Diabetes Federation. Diabetes atlas. (available at: http://www.diabetesatlas.org/resources/2017-atlas.html)

3 Brown S, Kim M, Mitchell C \& Inskip H. Twenty-five year mortality of a community cohort with schizophrenia. British Journal of Psychiatry 2010196 116-121. (https://doi.org/10.1192/bjp. bp.109.067512)

4 McCreadie R, Macdonald E, Blacklock C, Tilak-Singh D, Wiles D, Halliday J \& Paterson J. Dietary intake of schizophrenic patients in Nithsdale, Scotland: case-control study. BMJ 1998317 784-785. (https://doi.org/10.1136/bmj.317.7161.784)

5 Schizophrenia. Problems and disorders. Royal College of Psychiatry, 2015. (available at: http://www.rcpsych.ac.uk/healthadvice/ problemsdisorders/schizophrenia.aspx)

6 Tiihonen J. 11-year follow up of motality in patients with schizophrenia: a population based cohort study. Lancet 2009374 620-627. (https://doi.org/10.1016/S0140-6736(09)60742-X)

7 Holt RIG \& Mitchell A. Diabetes mellitus and severe mental illness: mechanisms and clinical implications. Nature Reviews Endocrinology 201511 79-89. (https://doi.org/10.1038/nrendo.2014.203)

8 Davy V, Wampers M, Mitchell AJ, Correll CU, De Herdt A, Probst M $\&$ De Hert M. A meta-analysis of cardio-metabolic abnormalities in drug naïve, first-episode and multi-episode patients with schizophrenia versus general population controls. World Psychiatry 201212 240-250.

9 Mitchell AJ. Is the prevelance of metabolic syndrome and metabolic abnormalities increased in early schizophrenia? A comparative metaanylsis of first epsiode, untreated and treated patients. Schizophrenia Bulletin 201339 295-305. (https://doi.org/10.1093/schbul/sbs082)

10 Wildgust $\mathrm{H} \&$ Beary M. Are there modifiable risk factors which will reduce the excess mortality in schizophrenia? Journal of Psychopharmacology 2010 37-50. (https://doi. org/10.1177/1359786810384639)

11 Smith M, Hopkins D, Peveler RC, Holt RI, Woodward M \& Ismail K. First- v. second-generation antipsychotics and risk for diabetes in schizophrenia: systematic review and meta-analysis. British Journal of Psychiatry 2008192 406-411. (https://doi.org/10.1192/bjp. bp.107.037184)

12 Rummel-Kluge C, Komossa K, Schwarz S, Hunger H, Schmid F, Lobos CA, Kissling W, Davis JM \& Leucht S. Head-to-head comparisons of metabolic side effects of second generation antipsychotics in the treatment of schizophrenia: a systematic review and meta-analysis. Schizophrenia Research 2010123 225-233. (https:// doi.org/10.1016/j.schres.2010.07.012)

13 Albaugh VL. A double blind, placebo controlled, randomized crossover study of the acute metabolic effects of olanzapine in health volunteers. PLOS ONE 20116 e22662. (https://doi.org/10.1371/ journal.pone.0022662)

14 Hirsch L, Yang J, Bresee L, Jette N, Patten S \& Pringsheim T. Secondgeneration antipsychotics and metabolic side effects: a systematic review of population-based studies. Drug Safety 201740 771-781. (https://doi.org/10.1007/s40264-017-0543-0)

15 Bhattacharjee J \& El-Sayeh HG. Aripiprazole versus typicals for schizophrenia. Cochrane Database of Systematic Reviews 2008 CD006617. (https://doi.org/10.1002/14651858.CD006617.pub3)

16 Citrome L, Kalsekar I, Baker RA \& Hebden T. A review of real-world data on the effects of aripiprazole on weight and metabolic outcomes in adults. Current Medical Research and Opinion 201430 1629-1641. (https://doi.org/10.1185/03007995.2014.908280)

17 Lieberman M. Effectiveness of antipsychotic drugs in patients with chronic schizophrenia. New England Journal of Medicine 2005353 1209-1223. (https://doi.org/10.1056/NEJMoa051688)
18 Kahn PRS. Effectiveness of antipsychotic drugs in first-episode schizophrenia and schizophreniform disorder: an open randomised clinical trial. Lancet 2008371 1085-1097. (https://doi.org/10.1016/ S0140-6736(08)60486-9)

19 Hammerman A. Antipsychotics and diabetes: an age-related association. Pharmacotherapy 200842 1316-1322. (https://doi. org/10.1345/aph.1L015)

20 Ronsley R, Nguyen D, Davidson J \& Panagiotopoulos C. Increased risk of obesity and metabolic dysregulation following 12 months of second-generation antipsychotic treatment in children: a prospective cohort study. Canadian Journal of Psychiatry 201560 441-450. (https://doi.org/10.1177/070674371506001005)

21 Galling B, Roldan A, Nielsen RE, Nielsen J, Gerhard T, Carbon M, Stubbs B, Vancampfort D, De Hert M, Olfson M et al. Type 2 diabetes mellitus in youth exposed to antipsychotics: a systematic review and meta-analysis. JAMA Psychiatry 201673 247-259. (https://doi. org/10.1001/jamapsychiatry.2015.2923)

22 Bobo WV. More evidence of an association between antipsychotic drugs and the risk of diabetes in children and adolescents: a population-based study. Evidence-Based Mental Health 201518 (https://doi.org/10.1136/eb-2014-101997)

23 Sagreiya H. Differences in antipsychotic related adverse events in adukt, paediatric and geriatric populations. Cureus 20179 e1059.

24 Bobo WV. Antipsychotics and the risk of type 2 diabetes mellitus in children and youth. JAMA Psychiatry 201370 1067-1075. (https:// doi.org/10.1001/jamapsychiatry.2013.2053)

25 National Institute for Health and Care Excellence. Psychosis and schizophrenia in children and young people: recognition and management, 2013. (available at: https://www.nice.org.uk/Guidance/ CG155)

26 Correll CU. Cardiometabolic risk of second generation antipsychotic medications durimng first time use in children and adolsecents. JAMA 2009302 1765-1773. (https://doi.org/10.1001/ jama.2009.1549)

27 Srisawasdi P, Vanwong N, Hongkaew Y, Puangpetch A, Vanavanan S, Intachak B, Ngamsamut N, Limsila P, Sukasem C \& Kroll MH. Impact of risperidone on leptin and insulin in children and adolescents with autistic spectrum disorders. Clinical Biochemistry 201750 678-685. (https://doi.org/10.1016/j.clinbiochem.2017.02.003)

28 Correll CU, Detraux J, De Lepeleire J \& De Hert M. Effects of antipsychotics, antidepressants and mood stabilizers on risk for physical diseases in people with schizophrenia, depression and bipolar disorder. World Psychiatry 201514 119-136. (https://doi. org/10.1002/wps.20204)

29 Vancampfort D, Correll CU, Galling B, Probst M, De Hert M, Ward PB, Rosenbaum S, Gaughran F, Lally J \& Stubbs B. Diabetes mellitus in people with schizophrenia, bipolar disorder and major depressive disorder: a systematic review and large scale metaanalysis. World Psychiatry 201615 166-174. (https://doi.org/10.1002/ wps.20309)

30 Barnett S, Meyers M, Alastair J, Flint M, Anthony J, Rothschild M, Benoit $\mathrm{H}$, Mulsant M, Ellen M, Whyte $\mathrm{M}$ et al. A double-blind randomized controlled trial of olanzapine plus sertraline vs olanzapine plus placebo for psychotic depression. Archives of General Psychiatry 200966 838-847. (https://doi.org/10.1001/ archgenpsychiatry.2009.79)

31 Chien IC, Wu EL, Lin CH, Chou YJ \& Chou P. Prevalence of diabetes in patients with major depressive disorder: a population-based study. Comprehensive Psychiatry 201253 569-575. (https://doi. org/10.1016/j.comppsych.2011.06.004)

32 Barnard K, Peveler R \& Holt RIG. Antidepressant medication as a risk factor for type 2 diabetes and impaired glucose regulation. Diabetes Care 201336 3337-3345. (https://doi.org/10.2337/dc13-0560)

33 Serretti J \& Mandelli L. Antidepressants and body weight: a comprehensive review and meta-analysis. Clinical Psychiatry 201071 1259-1272. (https://doi.org/10.4088/JCP.09r05346blu) 
34 National Institute for Health and Care Excellence. Dementia: supporting people with dementia and their carers in health and social care, 2006. (available at: https://www.nice.org.uk/guidance/ $\operatorname{cg} 42)$

35 Atti AR, Ferrari Gozzi B, Zuliani G, Bernabei V, Scudellari P, Berardi D, De Ronchi D, Tarricone I \& Menchetti M. A systematic review of metabolic side effects related to the use of antipsychotic drugs in dementia. International Psychogeriatrics 201426 19-37.

36 Albert SG, Grossberg GT, Thaipisuttikul PJ, Scouby J \& Green E. Atypical antipsychotics and the risk of diabetes in an elderly population in long-term care: a retrospective nursing home chart review study. Journal of the American Medical Directors Association 2009 10 115-119. (https://doi.org/10.1016/j.jamda.2008.08.005)

37 Zheng L, Mack W, Dagerman KS. Hsiao JK, Lebowitz BD, Lyketsos CG, Stroup TS, Sultzer DL, Tariot PN, Vigen C et al. Metabolic changes associated with second-generation antipsychotic use in alzheimer's disease patients: the CATIE-AD study. American Journal of Psychiatry 2009166 583-590. (https://doi.org/10.1176/ appi.ajp.2008.08081218)

38 Balkau B. Predicting diabetes: clinical, biological, and genetic approaches: data from the Epidemiological Study on the Insulin Resistance Syndrome (DESIR). Diabetes Care $2008312056-2061$. (https://doi.org/10.2337/dc08-0368)

39 Holt RIG \& Peveler R. Obesity, serious mental illness and antipsychotic drugs. Diabetes, Obesity and Metabolism 200911 665-679. (https://doi.org/10.1111/j.1463-1326.2009.01038.x)

40 Haupt D. Adiposity and insulin sensitivity derived from intravenous glucose tolerance tests in antipsychotic-treated patients. Neuropsychopharmacology 200832 2561-2569. (https://doi. org/10.1038/sj.npp.1301392)

41 Fernandez-Egea E. Metabolic effects of olanzapine in patients with newly diagnosed psychosis. Journal of Clinical Psychopharmacology 201131 154-159. (https://doi.org/10.1097/JCP.0b013e31820fcea3)

42 Starrenburg FCJ \& Bogers JP. How can antipsychotics cause diabetes mellitus? Insights based on receptor-binding profiles, humoral factors and transporter proteins. European Psychiatry $20092 \mathbf{4}$ 164-170. (https://doi.org/10.1016/j.eurpsy.2009.01.001)

43 Wang T, Li M \& Sun J. Circulating prolactin associates with diabetes and impaired glucose regulation. Diabetes Care 201336 1974-1980. (https://doi.org/10.2337/dc12-1893)

44 Miron IC, Baroana VC, Popescu F \& Ionica F. Pharmacological mechanisms underlying the association of antipsychotics with metabolic disorders. Current Health Sciences Journal 201440 12-17.

45 Reynolds GP \& Kirk SL. Metabolic side effects of antipsychotic drug treatment - pharmacological mechanisms. Pharmacology and Therapeutics 2010125 169-179. (https://doi.org/10.1016/j. pharmthera.2009.10.010)

46 Freyberg Z \& McCarthy MJ. Dopamine D2 receptors and the circadian clock reciprocally mediate antipsychotic drug-induced metabolic disturbances. NPJ Schizophrenia 20173 17. (https://doi. org/10.1038/s41537-017-0018-4)

47 Kaushal J, Bhutani G \& Gupta R. Comparison of fasting blood sugar and serum lipid profile changes after treatment with atypical antipsychotics olanzapine and risperidone. Singapore Medical Journal 201253 488-492.

48 Ballon J. Molecular pathophysiology of metabolic effects of antipsychotic medications. Cell Press 201425 593-600.

49 Vuk A, Kuzman MR, Baretic M \& Osvatic MM. Diabetic ketoacidosis associated with antipsychotic drugs: case reports and a review of literature. Psychiatria Danubina 201729 121-135. (https://doi. org/10.24869/psyd.2017.121)

50 Polcwiartek C. Diabetic ketoacidosis and diabetes associated with antipsychotic exposure among a previously diabetes-naive popoulation with schizophrenia: a nationwide nested case-control study. Diabetologia 201760 1678-1690. (https://doi.org/10.1007/ s00125-017-4320-5)
51 Citrome L \& Yeomans D. Do guidelines for severe mental illness promote physical health and well-being? Journal of Psychopharmacology 200519 102-109. (https://doi. org/10.1177/0269881105059505)

52 Mulligan K. Barriers and enablers of type 2 diabetes self-management in people with severe mental illness. Health Expectations 201720 1020-1030. (https://doi.org/10.1111/hex.12543)

53 The management of diabetes in adults and children with psychiatric disorders in inpatient settings. Joint British Diabtes Society-Inpatient and RCPsych Joint Guidelines, 2017. (https://www.diabetes.org.uk/ resources-s3/2017-10/Management\%20of\%20diabetes\%20in\%20 adults $\% 20$ and $\% 20$ children $\% 20$ with $\% 20$ psychiatric $\% 20$ disorders $\% 20$ in\%20inpatient\%20settings-August-2017.pdf)

54 Barnett A. Minimising metabolic and cardiovascular risk in schizophrenia: diabetes obesity and dyslipidaemia. Psychopharmacology 200721 357-373. (https://doi. org/10.1177/0269881107075509)

55 De Hert M. Cardiovascular disease and diabetes in people with severe mental illness position statement form the European Pyschiatry Association (EPA), supported by the European Association for the study of Diabetes (EASD) amd the European Society of Cardiology (ESC). European Psychiatry 200924 412-424. (https://doi. org/10.1016/j.eurpsy.2009.01.005)

56 Gothefors D. Swedish clinical guidelines -prevention and management of metabolic risk in patients with severe psychiatric disorders. Nordic Journal of Psychiatry 201064 294-302. (https://doi. org/10.3109/08039488.2010.500397)

57 Cooper SJ, Reynolds GP with Expert co-authors BAP guidelines on the management of weight gain, metabolic disturbances and cardiovascular risk associated with psychosis and antipsychotic drug treatment . Journal of Psychopharmacology 201630 717-748. (https:// doi.org/10.1177/0269881116645254)

58 Romain AJ, Letendre E, Akrass Z, Avignon A, Karelis AD, Sultan A \& Abdel-Baki A. Can HbA1c be used to screen for glucose abnormalities among adults with severe mental illness? Experimental and Clinical Endocrinology and Diabetes 2017125 251-255. (https://doi. org/10.1055/s-0042-116313)

59 Taylor J, Stubbs B, Hewitt C, Ajjan RA, Alderson SL, Gilbody S, Holt RIG, Hosali P, Hughes T \& Kayalackakom T. The effectiveness of pharmacological and non-pharmacological interventions for improving glycaemic control in adults with severe mental llness: a systematic review and meta-analysis. PLOS ONE 201712 e0168549. (https://doi.org/10.1371/journal.pone.0168549)

60 Caemmerer J, Correll CU \& Maayan L. Acute and maintenance effects of non-pharmacologic interventions for antipsychotic associated weight gain and metabolic abnormalities: a meta-analytic comparison of randomized controlled trials. Schizophrenia Research 2012140 159-168. (https://doi.org/10.1016/j.schres.2012.03.017)

61 Naslund JA, Whiteman KL, McHugo GJ, Aschbrenner KA, Marsch LA $\&$ Bartels SJ. Lifestyle interventions for weight loss among overweight and obese adults with serious mental illness: a systematic review and meta-analysis. General Hospital Psychiatry 201747 83-102. (https://doi.org/10.1016/j.genhosppsych.2017.04.003)

62 Daumit GL, Dickerson FB, Wang NY, Dalcin A, Jerome GJ, Anderson CA, Young DR, Frick KD, Yu A \& Gennusa JV 3rd. A behavioral weight-loss intervention in persons with serious mental illness. New England Journal of Medicine 2013368 1594-1602. (https://doi.org/10.1056/NEJMoa1214530)

63 Green CA, Yarborough BJ, Leo MC, Yarborough MT, Stumbo SP, Janoff SL, Perrin NA, Nichols GA \& Stevens VJ. The STRIDE weight loss and lifestyle intervention for individuals taking antipsychotic medications: a randomized trial. American Journal of Psychiatry 2015 172 71-81. (https://doi.org/10.1176/appi.ajp.2014.14020173)

64 Speyer H, Christian Brix Norgaard H, Birk M, Karlsen M, Storch Jakobsen A, Pedersen K, Hjorthøj C, Pisinger C, Gluud C, Mors O et al. The CHANGE trial: no superiority of lifestyle coaching plus 
care coordination plus treatment as usual compared to treatment as usual alone in reducing risk of cardiovascular disease in adults with schizophrenia spectrum disorders and abdominal obesity. World Psychiatry 201615 155-165. (https://doi.org/10.1002/wps.20318)

65 Hasnain M \& Victor W. Metformin for atypical antipsychoticinduced weight gain and glucose metabolism dysregulation. CNS Drugs 201024 193-206.

66 Ishøy PL, Knop FK, Broberg BV, Bak N, Andersen UB, Jørgensen NR, Holst JJ, Glenthøj BY \& Ebdrup BH. Effect of GLP-1 receptor agonist treatment on body weight in obese antipsychotic-treated patients with schizophrenia: a randomized, placebo-controlled trial. Diabetes, Obesity and Metabolism 201719 162-171.

67 Larsen JR, Vedtofte L, Jakobsen MSL, Jespersen HR, Jakobsen MI, Svensson CK, Koyuncu K, Schjerning O, Oturai PS, Kjaer A et al. Effect of liraglutide treatment on prediabetes and overweight or obesity in clozapine- or olanzapine-treated patients with schizophrenia spectrum disorder: a randomized clinical trial.
JAMA Psychiatry 201774 719-728. (https://doi.org/10.1001/ jamapsychiatry.2017.1220)

68 Stroup TS, Byerly MJ, Nasrallah HA, Ray N, Khan AY, Lamberti JS, Glick ID, Steinbook RM, McEvoy JP \& Hamer RM. Effects of switching from olanzapine, quetiapine, and risperidone to aripiprazole on 10-year coronary heart disease risk and metabolic syndrome status: results from a randomized controlled trial. Schizophrenia Research 2013146 190-195. (https://doi.org/10.1016/j. schres.2013.01.013)

69 Inzucchi SE, Bergenstal RM \& Buse JB. Position statement on management of hyperglycemia in type 2 diabetes American Diabetes Association and European Association for the Study of Diabetes. Diabetes Care 2015 140-149. (https://doi.org/10.2337/dc14-2441)

70 Foley D \& Mackinnon A. Effect of age, family history of diabetes and antipsychotic drug treatment on risk of diabetes in people with psychosis: a population based cross sectional study Lancet Psychiatry 20153 104-105. (https://doi.org/10.1016/S2215-0366(16)00004-3)

Received 9 January 2018

Revised version received 12 March 2018

Accepted 15 March 2018 